\title{
Introduction. Mode(s) et modalité(s) I
}

\section{(2) OpenEdition}

\section{Journals}

Édition électronique

URL : https://journals.openedition.org/ml/224

DOI : $10.4000 / \mathrm{ml} .224$

ISSN : 2274-0511

\section{Éditeur}

Association Modèles linguistiques

\section{Édition imprimée}

Date de publication : 1 juillet 2010

Pagination : 7-8

\section{Référence électronique}

«Introduction. Mode(s) et modalité(s) I », Modèles linguistiques [En ligne], 62 | 2010, mis en ligne le 09 janvier 2013, consulté le 01 juillet 2021. URL : http://journals.openedition.org/ml/224 ; DOI : https:// doi.org/10.4000/ml.224 


\section{Introduction}

\section{Mode(s) et modalité(s)}

I

Mode et modalité sont aujourd'hui deux concepts couramment utilisés en linguistique. Mais ce ne sont pas les seuls dans la série de termes hérités du latin modus. Dérivés de modus on trouve en latin, à différentes époques : le diminutif modulus, qui a donné fr. modèle, via l'italien modello (cf. aussi fr. moule et module) ; le verbe modulor/ari ("mesurer, soumettre à une mesure, moduler des vers et les chanter, tirer une mélodie d'un instrument, faire vibrer la lyre en accord avec le chant) ; sur modulor, une série de noms (modulatio, modulator/modulatrix, modulamen, modulamentum) ; deux adjectifs : modulatus, modulabilis ("harmonieux, mélodieux"), et un adverbe : modulate.

Outre mode, attesté depuis le XVe siècle, modèle (XVI) moule (XVI mais dès le $\mathrm{XI}^{\mathrm{e}}$ sous la forme modle) et module (XVIe), le français en a tiré ou développé pour son propre compte : modal et modalité (XVIe) moduler, modulation (fin $\mathrm{XV}$, mais rares avant le $\mathrm{XVII} e$ ), puis, plus récemment $\left(\mathrm{XX}^{\mathrm{e}}\right.$ s.), pour s'en tenir au domaine linguistique, modaliser et modalisation (modalisateur, modalisant) qu'enregistre le Trésor de la langue française, mais pas le Petit Robert.

À l'origine de cette famille de termes, l'idée centrale de modus est celle de "mesure" en général, d'où, dans le domaine de la musique et du chant, "cadence, rythme", mais aussi, par extension, ce que la cadence mesure, c'est-à-dire la "mélodie". Mode, au sens grammatical, apparaît au XVI siècle pour désigner une catégorie du verbe avec son contenu de «temps ».

Autour de ce foisonnement de termes et de concepts, Modèles linguistiques a organisé à Toulon en novembre 2007, dans le cadre de l'équipe d'accueil Babel, un colloque intitulé « Mode(s) et modalité(s) ».

Il était proposé d'articuler ce colloque sur trois volets :

$1^{\circ}$ Histoire critique des théories,

$2^{\circ}$ Études de divers systèmes modaux,

$3^{\circ}$ Analyses contrastives et « traductologie ». 
Les langues retenues étaient quelques-unes de celles qui sont parlées sur le pourtour et dans les îles de la Méditerranée, en l'occurrence l'arabe, le berbère, le catalan, l'espagnol, le français, l'italien et, bien entendu l'anglais, une des deux langues officielles de Malte, dont l'importance géographique, historique et stratégique n'est plus à démontrer.

Modèles linguistiques publie en trois volumes (62, 63 et 64) les communications remaniées qui ont été présentées à ce colloque. L'ordre de publication n'est pas celui du triptyque donné ci-dessus. Le présent volume illustre les second et troisième volets, avec des analyses en synchronie contemporaine de divers systèmes de modalités dans les langues mentionnées plus haut. La moitié des articles de ce volume adoptent un point de vue contrastif.

Les deux numéros suivants (vols 63 et 64) seront consacrés au premier volet du triptyque : « Histoire critique des théories ». Le volume 63, Mode(s) et modalité(s) II (tome XXXII-1, 2011) opérera une remontée dans le temps. On y trouvera une série d'études centrées sur le français et partiellement sur l'anglais, du XVIe siècle à la fin du XIXe.

Enfin Mode(s) et modalité(s) III (tome XXXII-2, 2011) traitera du mode et de la modalité de la fin du XIXes. (Bréal) à nos jours.

Modèles linguistiques 